\title{
Risk factors associated with metabolic syndrome by enriched food in pig fat in the Wistar rats
}

\begin{abstract}
Background: The metabolic syndrome is a state of morbidity characterized by factors such as abdominal obesity, high blood pressure, hyperglycemia, low HDL cholesterol (HDL-C) and high triglyceride levels. The objective of this study was to evaluate the effect of a diet supplemented with pig fat $10 \%$ and $30 \%$ on the occurrence of at least three of the five factors that characterize metabolic syndrome with a model animal imitating the pathological state of man.
\end{abstract}

Material and methods: The experiment was conducted on three groups of Wistar rats (Rattus norvegicus) ad libitum consumption pig high-fat diet (HF, 30\%), pig lowerfat diet (LF, 10\%) and normal diet (ND) for 9 weeks. At the end of the experiment, metabolic parameters were obtained and adipose tissue was weighted.

Results: The results showed that total cholesterol was significantly increased in rats consuming $10 \%$ and $30 \%$ fat compared to control rats $(142.44 \pm 5.3$ and $148.86 \pm 4.25$ vs $136.02 \pm 4.8 \mathrm{mg} / \mathrm{dl} ; \mathrm{p}<0.05 ; \mathrm{n}=7$ ). It's the same for blood triglycerides concentration in experimental rats (LF, HF) and control rats with values of $128.25 \pm 4.7$ and $129.02 \pm 5.4$ vs $112,4 \pm 3.6 \mathrm{mg} / \mathrm{dl}(\mathrm{p}<0.05)$. The LDL cholesterol was singularly high in rats fed the $30 \% \mathrm{HF}$ diet compared to the rest of the animals $(112.8 \pm 8.5$ vs $96.40 \pm 9.3$ and $90.4 \pm 4.2$ $\mathrm{mg} / \mathrm{dl} ; \mathrm{p}<0.05)$. The serum fraction of HDL cholesterol was significantly lower in the experimental rats $(\mathrm{LF}, \mathrm{HF})$ than the control rats $(61 \pm 4.9$ and $54.97 \pm 5.9$ vs $93.5 \pm 4.1$ $\mathrm{mg} / \mathrm{dl} ; \mathrm{p}<0.05)$. The atherogenic indices CT/HDL and LDL/HDL were significantly higher in LF and HF rats compared to control rats (1.78 and 1.52 vs $1.34 ; p<0.05)$. The plasma glucose level of the experimental rats (LF, HF) was significantly reduced compared to that of the control rats $(23.3 \pm 3.21$ and $25.7 \pm 2.4$ vs $34.7 \pm 3.04 \mathrm{mg} / \mathrm{dl})$ $(p<0,05)$. The percentage of epididymal adipose tissue is also greater in diet animals (HF) than standard (RS) and experimental (LF) rats $(1.4 \pm 0.30 \%$ vs. $0.79 \pm 0,06 \%$ and $0.75 \pm 0.04 \% ; \mathrm{p}<0.05$ )

Conclusion: Elevated levels serum of total cholesterol, LDL cholesterol and atherogenic indices (TC/HDL and LDL/HDL), low HDL-C and excessive accumulation of adipose tissue (abdominal and epidydimal) have shown that the standard diet supplemented with $10 \%$ or $30 \%$ of pig fat caused a metabolic syndrome. Like the animal fat, incorporation at high doses in the diet could affect health because of the presence of hyperLDLlemia found in our test which one were the main risk factors for cardiovascular disease.

Keywords: Biochimicals parameters, Fat diet, Adipose tissue, Metabolism syndrome, Wistar rat
Volume 5 Issue 6 - 2018

\author{
Monteomo GF, Kamagate A, Bleyere NM, \\ Yapo AP
}

Laboratory of Physiology, Pharmacology and Pharmacopoeia, UFR-Science of Nature, University in Abidjan, Côte d'Ivoire

\begin{abstract}
Correspondence: Monteomo Gnate Francois, Laboratory of Physiology, Pharmacology and Pharmacopoeia, UFR-Science of Nature, University in Abidjan, Côte d'Ivoire,

Emailmonteamo_gnate@yahoo.fr
\end{abstract}

Received: November 21, 2018 | Published: December 10
,2018

\section{Introduction}

Metabolic syndrome (SM) is also known as Syndrome $X,{ }^{1}$ is a morbid condition, characterized by an aggregate of cardiovascular risk factors and type 2 diabetes such as abdominal obesity, high blood pressure, high blood sugar, low HDL-cholesterol (HDL-C) and hypertriglyceridemia. ${ }^{2}$ A person has metabolic syndrome (MS) when he/she has a combination of 3 or more specific health risks. It's becoming an emerging global public health problem, global prevalence can be estimated at about a quarter of the world's population. In other words, more than one billion people worldwide are affected by the metabolic syndrome. ${ }^{3}$ This epidemic affects about $35 \%$ and $50 \%$ of the adult population respectively to United States and Northern Europe. ${ }^{4}$ The prevalence of MS varies by $4.4 \%$ in Côte d'Ivoire. ${ }^{5}$ The deleterious effects of MS draw research efforts in developing new interventions to reduce its burden on the healthcare system. Due to its multifactorial nature, selecting an adequate experimental model that best represents the path physiology of MS in humans can be rather challenging. Rats and mice are the most common animal models used in investigating MS. Some of the various approaches used to induce MS in rodents include dietary manipulation, genetic modification and drugs. ${ }^{6}$ Although heredity is one of the causes of this syndrome, the vast majority of cases is rather related to a sedentary lifestyle and a poor diet including the consumption of high carbohydrate and fat food that would cause lipid abnormalities which are atherogenic. ${ }^{7}$

Thus, several studies use diets enriched with fats of various origin for the induction of the metabolic syndrome in animal models developing symptoms as close as possible to those found in humans to test appropriate treatments. ${ }^{8,9}$ Among these, they are high fat diets lardy based (thick fat layer of subcutaneous tissue of pig) appears to be the most obesogenic with a composition predefined. ${ }^{10}$ Pig's fat is 
a melting fat that is characterized by a high content of saturated fatty acids (40 to 50\%) and a low concentration of linoleic acid (from 2 to $8 \%)$.

In addition, previous work done in our laboratory showed that a consumption of granules (FACI ${ }^{\circ}$, Côte d'Ivoire) supplemented by palm oil to $15 \%$ didn't both lead to weight gain and elevated cholesterol levels in Wistar rats. Of even, an absence the metabolism syndrome has been noted in these animals aged 8 to 9 after 4 weeks of testing. ${ }^{11}$

The objective of this work is to evaluate the effects of a normal diet supplemented with low dose of $10 \%$ (LF) and high dose of $30 \%$ (HF) pig fat on the appearance of metabolic syndrome factors in Wistar rats during a period of 9 weeks.

\section{Material and Methods}

\section{Animal material and livestock}

The experimental protocol consisted of 21 rats mixed in 3 groups of 7, as well as Wistar strain (Rattus norvegicus) composed of males and females, of the pet shop of the physiology, pharmacology and pharmacopoeia laboratory of the UFR-SN, Nangui Abrogoua University, Abidjan. Rats aged 8 to 9 weeks and with a homogeneous mean body weight of $110.60 \pm 7.5 \mathrm{~g}$ are fed either with pellets $(\mathrm{FACI} \AA$, Abidjan, Côte d'Ivoire) or to grains added to $10 \%$ (LF) and 30\% (HF) pig fat for experimental rats for 9 weeks (63 days). The breeding is done in a lighted room 12 hours a day, and whose temperature is kept constant $\left(22\right.$ to $\left.23^{\circ} \mathrm{C}\right)$ in an enclosure equipped with an air conditioner (Smart, Canada). The animals have free access to food and water and are weighed once a week.

\section{Technical material}

Technical material consists of electronic scale, reagent (alcohol, ether), anesthesia bell, cotton, Pasteur pipette, dry tubes and specimens.

\section{Collection of blood samples}

Blood samples were taken in healthy rats on days 0 and 56 . At each sample, the animals were fasted for 12 hours. In animals anesthetized with ether, blood was taken from the retro-orbital sinus using a sterile Pasteur pipette. Blood samples were collected in dry tubes. After centrifugation at $4000 \mathrm{rpm}$ for $20 \mathrm{~min}$, the serum is recovered for biochemical analyzes (triglycerides, total cholesterol, and HDLcholesterol and blood glucose).

\section{Biochemical analyzes}

Total cholesterol (TC), HDL-cholesterol (HDL-C), triglycerides (TG) and blood glucose are determined by the enzymatic and colorimetric method, ${ }^{12}$ using a Robonik Prietest (India). As for LDLcholesterol (LDL-C), it's calculated from the Friedwald formula below: $\mathrm{LDL}-\mathrm{C}=\mathrm{CT}-(\mathrm{HDL}-\mathrm{C}+\mathrm{TG}) / 5$

These determined biochemical parameters expressed (mg/dl) make it possible to calculate an atherogenicity index (CT/HDL or LDL/ HDL) which is a revealing indicator of the arterial and especially coronary risk. ${ }^{13}$

\section{Statistical analyzes}

The statistical analysis of the data is done with the Graph Pad Prism
5.01 software (San Diego, California, USA). The results are given as an average followed by the standard error on the mean $(M \pm S E M)$. The analysis of the variance is completed by the Turkey-Kramer test to compare the average values of the different parameters. Differences are considered significant in $\mathrm{P}<0.05$.

\section{Results}

After 9 weeks, the body weight gain ( $\mathrm{g}$ ) of LF rats was significantly higher than those ND rats and HF rats $(59.5 \pm 6.8 \mathrm{~g}$ vs $26.9 \pm 7.3 \mathrm{~g}$ and $30.8 \pm 4.6 \mathrm{~g} ; \mathrm{P}<0,05$ ) (Figure 1A) .

Similarly, body weight gain in the animal correlated with the amount of food ingested $(\mathrm{g} / \mathrm{d})$. Thus, the LF rats have consumed an average of $42,75 \pm 5,7 \mathrm{~g} /$ day while those of the normal group (ND) and experimental (HF) consumed respectively, $38.5 \pm 25$ and $27.5 \pm 3 \mathrm{~g} /$ day (Figure 1B).

The average weight relative adipose tissue (abdominal and epididymal) have been reported in percentage (\%) of body weight of rats following the animals dissection at the end of the experiment. The percentage of abdominal adipose tissue of HF rats was significantly higher than rats receiving normal (ND) and experimental LF diet $(3.5 \pm 0.9 \%$ vs $2.9 \pm 0.6 \%$ and $1.8 \pm 0.7 \% ; \mathrm{p}<0.05)$. The percentage of epididymal adipose tissue was also greater in HFrat than Normal (ND) and experimental (LF) rats $(1.4 \pm 0.30 \%$ vs $0.79 \pm 0,06 \%$ and $0.75 \pm 0.04 \%$; $<<0.05$ ) (Figs. 2 and 3). However, perirenal adipose tissue didn't show a significant difference between all groups of rats $(0.8 \pm 0.05 \%$ vs $0.7 \pm 0.08 \%$ and $0.75 \pm 0.04 \%$; $>>0.05)$.

The levels of triglycerides and lipoproteins (CT, LDL-C, and HDL-C) associated with their atherogenic index and blood glucose was recorded in the table above.
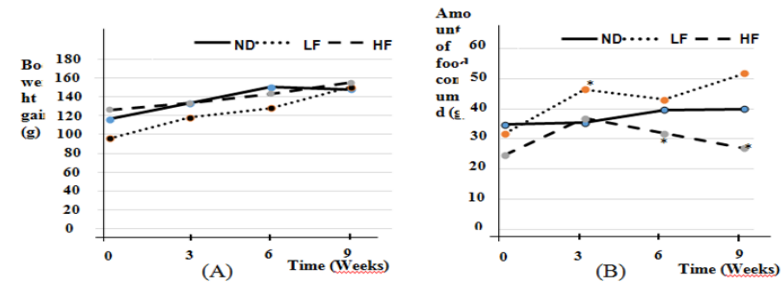

Figure I Body weight (A) and amount of food consumed (B) in Wistar rats fed food supplemented with $10 \%$ (LF) and $30 \%$ (HF) pig fat during 9 weeks. The data are presented on average \pm SEM $(n=7$ rats; $P<0.05)$. S Significative difference between Normal diet (ND) and diet groups supplemented with $10 \%(\mathrm{LF})$ or $30 \%(\mathrm{HF})$ pig fat.

\section{Discussion}

The consumption of the normal food supplemented with pig fat had various effects on metabolic syndrome components such as the body weight gain, adipose tissue, blood lipids associated to their atherogenic indices and metabolism carbohydrate.

The significant increase in body weight gain of LF rats $(\mathrm{p}<0.05$; $\mathrm{n}=7$ ) compared to other animals over a period of 9 weeks would be due to palatability high for this fat diet that has been relatively moderate. The underperformance of body weight gain in rats HF be explained by having a resistance to obesity following an adaptation of animals to fatty diets or a reduction of food consumption (Figure 1B). ${ }^{14}$

In addition, the significant increase in the percentage of epididymal 
and abdominal adipose tissue was observed in HF rats compared to the rest of the rats $(p<0.05)$ (Figure $2 \&$ Figure 3$)$ confirmed an abnormal development of adipose tissue synonymous with obesity, more predictive of metabolic syndrome in these animals although their body weight is not known to increase significantly.

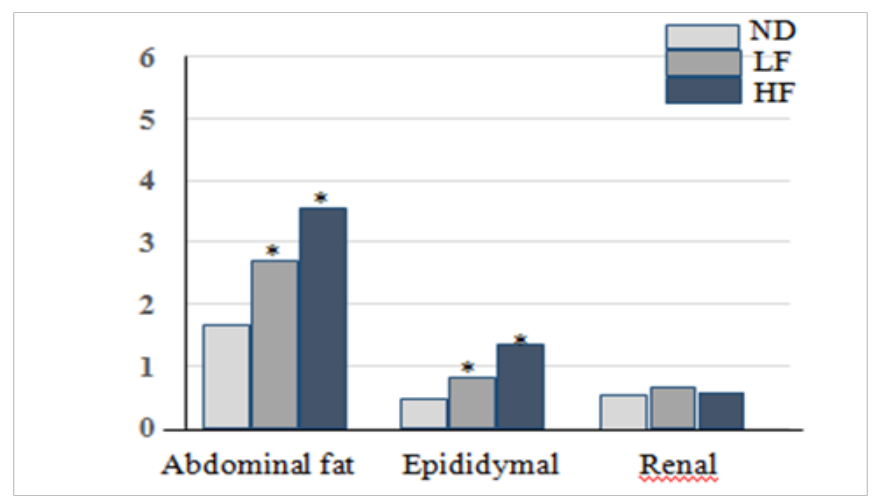

Figure 2 Abdominaux fats, epididymal and renal adipose tissue in Wistar rats at the end of the diet. The data are presented as mean $\pm S D(n=7$ rats, $\mathrm{P}<0.05)$ *: Significant difference between Normal diet (ND) and diet groups supplemented with $10 \%$ (LF) and 30\% (HF) pig fat.

The analysis of blood lipid composition showed that total cholesterol levels, LDL cholesterol and increased triglycerides levels very significantly in the experimental rats (HF and LF) compared to control rats $(\mathrm{p}<0.05 ; \mathrm{n}=7)$ (Table). In addition, the LDL cholesterol was singularly in HF rats. Indeed, the increase in triglyceride levels

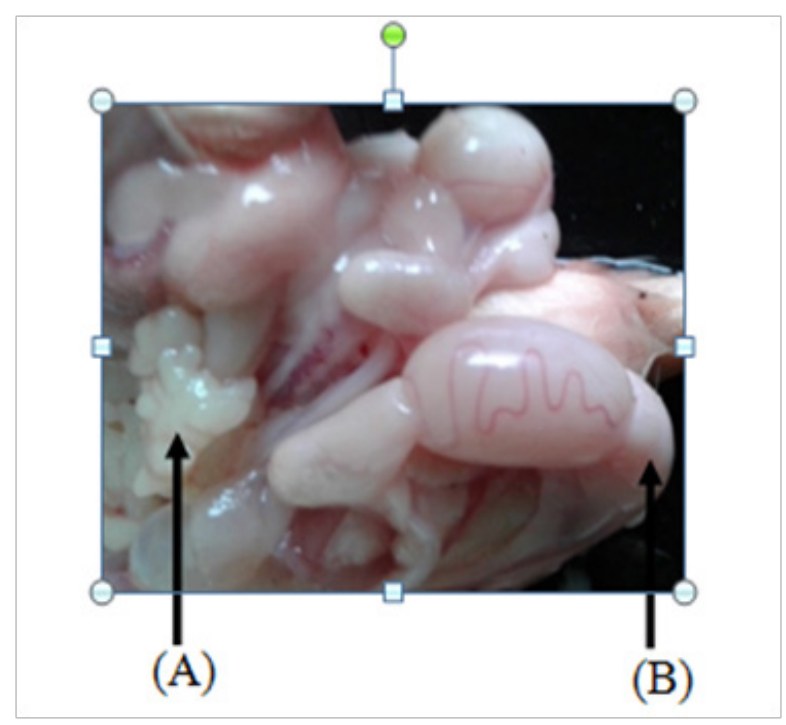

Figure 3 Abdominaux fats tissus (A) and Epididymal (B) in $\mathrm{HF}$ rats

and in particular LDL-C is a tool for assessing and monitoring the presence of the risk of coronary disease and atherosclerosis. ${ }^{15}$ The alteration of lipid metabolism already emphasized has also been found in other experiments with the fat doses, ${ }^{16}$ and were the main cause of MS regardless of obesity.

Table I Biochemical Parameters are Wistar rats fed various diets for 9 weeks. The data are presented as mean \pm SD $(n=7$ rats, $P<0.05) *:$ Significant difference between supplemented diet groups and Control group. HF, high fat diet; LF, low fat diet; ND, Normal diet (Control group). LDL-C, low-density lipoprotein cholesterol; HDL-C, high density lipoprotein cholesterol; CT, total cholesterol;TG, triglycerides; Ai,Atherogenic index.

\begin{tabular}{|c|c|c|c|c|c|c|}
\hline \multirow[b]{2}{*}{ Physiological parameters } & \multicolumn{3}{|c|}{ Initial concentrations (D0) } & \multicolumn{3}{|c|}{ Final concentrations (D 63) } \\
\hline & ND & LF ( $10 \%)$ & HF (30\%) & ND & LF (10\%) & HF (30\%) \\
\hline $\mathrm{TC}, \mathrm{mg} / \mathrm{dl}$ & $134,2 \pm 1.4$ & $131,5 \pm 1.66$ & $138,17 \pm 1.48$ & $136,02 \pm 4.8$ & $142.44 \pm 5.3$ & $148.86 \pm 4.25$ \\
\hline HDL-C, mg/dl & $90,5 \pm 5.4$ & $78.5 \pm 5.7$ & $91.1 \pm 4,8$ & $93,5 \pm 4.1$ & $61 \pm 4,9$ & $54,97 \pm 5.9 *$ \\
\hline LDL-C, mg/dl & $92.5 \pm 4.7$ & $90.1 \pm 4,1$ & $79.5 \pm 4,3$ & $90,4 \pm 4,2$ & $96.40 \pm 9.3 *$ & $112.8 \pm 8.5$ \\
\hline $\mathrm{TG}, \mathrm{mg} / \mathrm{dl}$ & $113.6 \pm 2.6$ & $109.8 \pm 1.8$ & $115,25 \pm 2.1$ & $112.4 \pm 3,6$ & $128.25 \pm 4,7$ & $129.02 \pm 5.4$ \\
\hline $\mathrm{Ai}=\mathrm{TG} / \mathrm{HDL}-\mathrm{C}$ & $1.25 \pm 0.48$ & $1.39 \pm 0.33$ & $1.26 \pm 0.55$ & $1.20 \pm 0.85$ & $2.09 \pm 0.95 *$ & $2.38 \pm 0.91 *$ \\
\hline$A i=L D L / H D L-C$ & $\mathrm{I}, 02 \pm 0,8$ & $\mathrm{I}, 15 \pm 0,7 \mathrm{I}$ & $0.86 \pm 0,8$ & $0.95 \pm 0,61$ & $1.57 \pm 0.55 *$ & $2.07 \pm 0.55 *$ \\
\hline Glucose, mg/dl & $33.7 \pm 1.33$ & $35.1 \pm 1.05$ & $31.9 \pm 1.12$ & $34.7 \pm 3.04$ & $23.3 \pm 3.21 *$ & $25.7 \pm 2.4 *$ \\
\hline
\end{tabular}

The mechanism of lipid metabolism dysfunction in the advent of the MS begins with the increase of the size; the number and the lipolytic activity of the adipocytes are at the origin of a significant release of triglycerides from the adipose tissue resulting at high levels of circulating fatty acids. Also, the presence of fat abdominal and epididymal fat (figure 3) that was reported in this experiment could aggravate the effects of excess adiposity by promoting a greater influx of fatty acids directly to the liver. This leads an increase in synthesis 
hepatic triglycerides and VLDL lipoproteins production and leads to long-time dyslipidemia, ${ }^{17}$ a sign of profound disturbance of lipid metabolism.

Conversely, the HDL cholesterol level was significantly higher in control rats than LF and HF rats $(93.5 \pm 4.1$ vs $61 \pm 4.9$ et $54.5 \pm 5.9$ $\mathrm{mg} / \mathrm{dl}$ ) in the table. This has been also noticed in rats fed a diet enriched with both animal and vegetable fat, giving a greater drop in HDL-C $(55,99 \pm 3.3$ vs $27.72 \pm 2 \mathrm{mg} / \mathrm{dl}){ }^{18}$ which will increased the risk of metabolic syndrome in animal11 and in humans. ${ }^{19}$ Indeed, HDL plays an important role in the reverse transport of cholesterol from peripheral tissues to the liver, ${ }^{20}$ but also exert direct actions on the vascular endothelium with, in particular, anti-inflammatory and antioxidant effects, ${ }^{21}$ delaying the onset of symptoms of metabolic syndrome as showed the animals receiving the normal diet.

The atherogenic index TC/HDL and LDL/HDL were significantly greater in HF and LF rats compared to control group (1.78 and $1.52 \mathrm{vs} .1 .34 ; \mathrm{p}<0,05)$, this is predictable the atherosclerosis. ${ }^{18,22}$ These atherogenic index to experimental rats are nevertheless lower at 5.6 obtained with a diet supplemented both fat and sugar, ${ }^{22}$ which involves high fatty acid by the mechanism already described.

In sum concerning cholesterol parameters, elevated serum TC, LDL-C and atherogenic index, low level of HDL-C and obesity were considered as a major risk factor for the development of the metabolic syndrome following chronic consumption of hyperlipidic diet in rodents and human. ${ }^{1,8,9}$

In terms of carbohydrate metabolism, against all odds, this hyperlipidic diet leads to a clear reduction in plasma glucose levels in LF and HF rats compared to control rats $(23.3 \pm 3.21$ and $25.7 \pm 2.4$ vs $34 \pm 3.04 \mathrm{mg} / \mathrm{dl} ; \mathrm{p}<0.05) .^{23}$ This could be attributed to a deficiency of glucose 6 phosphatase and the blocking of glycogenolysis then hepatic neoglucogenesis at the origin of moderate hypoglycaemia. ${ }^{24}$ This abnormal reduction in blood glucose occurs when there is a risk of insulin resistance and it could be the cause of metabolic dyslipidemia previously noted. ${ }^{25}$

\section{Conclusion}

The use of a diet supplemented with $10 \%$ pig fat in 9-weekold Wistar rats revealed three risk factors leading to the metabolic syndrome based on hypertriglyceridemia, hypercholesterolemia and low HDL. The remarkable increase of LDL in the animals subjected to the $30 \%$ pig fat in diet confirms at this dose its more atherogenic character compared to that $10 \%$. Added to this is a disruption of carbohydrate metabolism in animals with moderate hypoglycaemia. These results support the epidemiological data that suggest that a high fat diet promotes the development of the metabolic syndrome.

\section{Acknowledgment}

The author would like to thank the Masters students and doctoral of Laboratory of Physiology, Pharmacology and Pharmacopoeia, Nangui Abrogoua University to provide assistance to testing on laboratory animals.

\section{Conflicts of interest}

The author declares that there is no conflict interest.

\section{References}

1. Reaven GM. Role of insulin resistance in human disease Diabetes. 1988;37(12):1595-1607.
2. Definition of metabolic syndrome in definition, diagnosis and classification of diabetes mellitus and its complications. Part I: Diagnosis and classification of diabetes mellitus. World Health Organization. 1999;31-32.

3. Saklayen MG. The global epidemic of the metabolic syndrome. Curr Hypertens Rep. 2018;20(12):1-8.

4. Aguilar M, Taft Bhuket, Sharon Torres, et al. Prevalence of the metabolic syndrome in the United States, 2003-2012. JAMA. 2015;313(19):1973.

5. Hauhouot AML, Yayo SE, Ake-Edjeme A, et al. Le syndrome metabolique existe-t-il en Côte d'Ivoire ?. Immuno-analyse \& Biologie Specialisee. 2008;23(6):375-378.

6. Wong SK, Kok YC, Suhaimi HF, et al. Animal models of metabolic syndrome: a review. Nutrition \& Metabolism. 2016;13(65):1-12.

7. Vergès B. Pathophysiology of dyslipidemia in patients with metabolism syndrome or type 2 diabetes mellitus. Nutr Clin Metab. 2007;21(1):9-16.

8. Roberts CK, Vaziri ND, Liang $\mathrm{KH}$, et al. Reversibility of chronic experimental syndrome $\mathrm{X}$ by diet modification. Hyperten. 2001;37,1323-1328.

9. Dobrian AD, Davies MJ, Schriver SD, et al. Oxidative stress in a rat model of obesity-induced hypertension. Hyperten. 2001;37,554-560.

10. Buettner R, Scholmerich J, Bollheimer LC. Modeling the metabolic disorders of human obesity in rodents. Obesity. 2007;15(4):798-808.

11. Bogue AIH, Kamagate A, Angoue YP. Prevalence à l'obesite par la consommation moderee d'huile de palme chez le rat. European Scientific Journal. 2016;12(30):224-242.

12. Talke H, Schubert GE. Enzymatic urea determination in the blood and serum in the Warburg optical test. Klin Wochenschr. 1965;43:174-175.

13. Niroumand Sh, Khajedaluee M, Khadem M, et al. Atherogenic index of plasma (AIP): a marker of cardiovascular disease. Med J Islam Repub Iran. 2015;29(240):1-9.

14. Bessesen DH, Bull S, Marc A. Trafficking of dietary fat and resistance to obesity. Physiol Behav. 2008;94(5):681-688.

15. Rastogi R, Reddy, Vaz Spieldman. Diet and risk of ichemic heart disease in India. Am J Clin Nutr. 2004;79:582-592.

16. Axen KV, Harper MA, Kuo YF, et al. Very low-carbohydrate, high-fat, weight reduction diet decreases hepatic gene response to glucose in obese rats. Nutr \& Metab. 2018;15(54):1-13.

17. Lewis GF, Carpentier A, Adeli K, et al. Disordered fat storage and mobilization in the pathogenesis of insulin resistance and type 2 diabetes. Endocrine reviews. 2002;23;201-229.

18. Saadah NN, Purwani KI, Nurhayati APD, et al. Analysis of lipid profile and atherogenic index in hyperlipidemic rat (Rattus norvegicus Berkenhout, 1769) that given the methanolic extract of Parijoto (Medinilla speciosa). AIP Publishing. 2017;1-8

19. Vafa M, Haghighatjoo E, Ziaee A. Effect of apple consumption on lipid profile of Hyperlipidemic and Overweight Men. Inter J Prev Med. 2011;2(2):84-100.

20. Farrer S. Beyond statins: Emerging evidence for HDL-increasing therapies and diet in treating cardiovascular disease. Adv Prev Med. 2018:1-9.

21. Auberval N, Dal S, Bietiger W, et al. Metabolic and oxidative stress markers in Wistar rats after 2 months on a high-fat diet. Diabetology \& Metabolic Syndrome. 2014;6(130):1-9.

22. Temme EH, Van HP, Schouten EG, et al. Effects of a plant sterol-enriched spread on serum lipids and lipoproteins in mildly hypercholesterolaemic subjects. Acta Cardiol. 2002;57:111-115.

23. Nasri R, Abdelhedi O, Jemil I, et al. Preventive effect of goby fish protein hydrolysates on hyperlipidemia and cardiovascular disease in Wistar rats fed a high-fat/fructose diet. $R S C A d v .2018 ; 8,93-83$. 
24. Bayraktar Y. Glycogen storage diseases: New perspectives. World J 25. Ensling M, Steinmann W, Whaley CA. Hypoglycemia: A possible link Gastroenterol. 2007;13(18):2541-2553. between insulin resistance, metabolic dyslipidemia and heart and kidney disease (the cardiorenal syndrome). Cardiorenal Med. 2011;1(1):67-74. 